\title{
On Design of Collaborative Beamforming for Two-Way Relay Networks
}

\author{
Meng Zeng, Rui Zhang, and Shuguang Cui
}

\begin{abstract}
We consider a two-way relay network, where two source nodes, S1 and S2, exchange information through a cluster of relay nodes. The relay nodes receive the sum signal from S1 and S2 in the first time slot. In the second time slot, each relay node multiplies its received signal by a complex coefficient and retransmits the signal to the two source nodes, which leads to a collaborative two-way beamforming system. By applying the principle of analog network coding, each receiver at S1 and S2 cancels the "self-interference" in the received signal from the relay cluster and decodes the message. This paper studies the 2-dimensional achievable rate region for such a two-way relay network with collaborative beamforming. With different assumptions of channel reciprocity between the source-relay and relay-source channels, the achievable rate region is characterized under two setups. First, with reciprocal channels, we investigate the achievable rate regions when the relay cluster is subject to a sum-power constraint or individual-power constraints. We show that the optimal beamforming vectors obtained from solving the weighted sum inverse-SNR minimization (WSISMin) problems are sufficient to characterize the corresponding achievable rate region. Furthermore, we derive the closed form solutions for those optimal beamforming vectors and consequently propose the partially distributed algorithms to implement the optimal beamforming, where each relay node only needs the local channel information and one global parameter. Second, with the non-reciprocal channels, the achievable rate regions are also characterized for both the sum-power constraint case and the individual-power constraint case. Although no closed-form solutions are available under this setup, we present efficient numerical algorithms by solving a sequence of semi-definite programming (SDP) problems after semi-definite relaxation (SDR), where the optimal beamformer can be obtained under the sum-power constraint and approximate optimal solutions can be obtained under the individual power constraints.
\end{abstract}

\section{Index Terms}

Two-Way Relay, Collaborative Beamforming, Achievable Rate Region, Pareto Optimal, Semi-definite Programming (SDP), Semi-definite Relaxation (SDR).

Meng Zeng and Shuguang Cui are with the Department of Electrical and Computer Engineering, Texas A\&M University, College Station, TX, 77843. Emails: \{zengm321, cui\}@tamu.edu.

Rui Zhang is with the Institute for Infocomm Research, A*STAR, Singapore and the Department of Electrical and Computer Engineering, National University of Singapore. Email: rzhang@i2r.a-star.edu.sg or elezhang@nus.edu.sg. 


\section{INTRODUCTION}

Cooperative communication has been extensively studied in past years, where various cooperative relaying schemes have been proposed, such as amplify-and-forward (AF) [1], decode-and-forward (DF) [2], compress-andforward (CF) [3], and coded-cooperation [4]. Among these schemes, due to its simplicity, the AF-based relaying is of the most practical interest, where multi-antenna relay beamforming has also been explored to achieve higher spatial diversity [5]. In certain resource constrained networks, such as sensor networks, the node size is limited such that each node could only mount a single antenna [6]. In order to exploit the multi-antenna gain in such sizelimited cases, collaborative relay beamforming strategies have been developed where the relaying nodes cooperate to generate a beam towards the receiver under sum or individual power constraints [7][8].

As an extension to the AF-based one-way relaying scheme, the AF-based two-way relaying scheme [9] is based on the principle of analog network coding (ANC) [10] to support communications in two directions. Traditionally, two-way relaying avoids the simultaneous transmissions of two source terminals, and requires four time-slots to finish one round of information exchange between them. On the contrary, the two-way relaying scheme proposed in [10] allows the relay to mix the data and amplify-and-forward it, where the two terminals exploit the underlying self-interference structure. By doing so, the amount of required transmission time-slots is reduced from four to two and the overall network throughput is thus improved. There are several other works discussing such two-way relay systems. In particular, the authors in [9] characterized the maximum achievable rate region for the two-way relay beamforming scheme by assuming a single relay node equipped with multiple antennas and two source nodes each equipped with a single antenna. As a counterpart of the work in [9], the decode-and-forward two-way relaying has been studied in [11] and the performance bounds are given in [12]. The authors in [13] studied the AF-based two-way relay with collaborative beamforming, where the focus is to minimize the total transmit power of the source nodes and the relay cluster under a given pair of signal-to-noise ratio (SNR) constraints.

The works on characterizing the rate region of two-way relaying has also been done in [14], where authors considered the collaborative beamforming case. However, all of existing works only obtained numerical solutions. The missing of closed-form solutions leads to difficulties in designing efficient algorithms due to the lack of insight into the structure of the optimal beamforming vectors. Thereby, in this paper, we try to seek the closed-form solutions for the optimal beamforming vectors to characterize the maximum achievable rate region and correspondingly propose efficient distributed algorithms. Our work differs from the work in [9] from two main aspects. First, we assume a cluster of single-antenna relay nodes and consider collaborative two-way relay beamforming rather than the multiple-antenna single-relay beamforming. Due to the distributed feature, we will study the case where each relay node has an individual power constraint in addition to the case where all relay nodes are subject to a sumpower constraint. Second, we present closed-form solutions for the optimal beamforming vectors when we have 


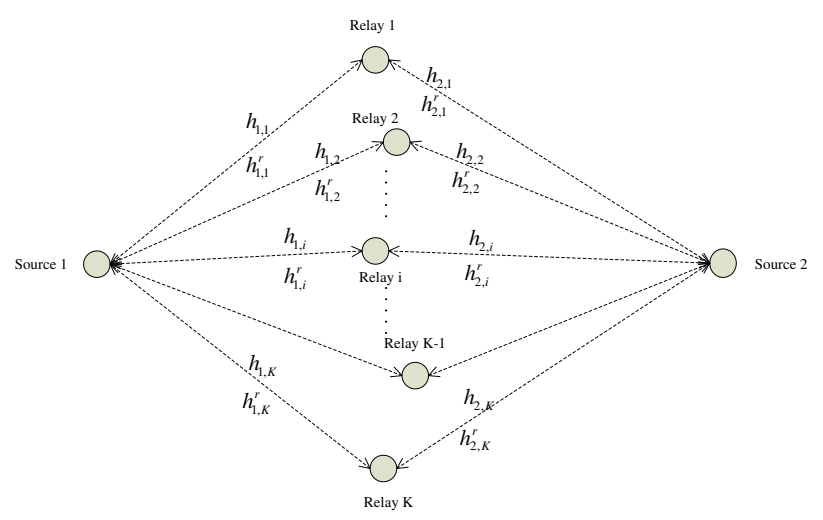

Fig. 1: System model

reciprocal channels. For the non-reciprocal channels, the achievable rate regions are also characterized. Although no closed-form solutions are available under this setup, we present efficient numerical algorithms by solving a sequence of semi-definite programming (SDP) problems after semi-definite relaxation (SDR), where the optimal beamformer can be obtained under the sum-power constraint and approximate optimal solutions can be obtained under the individual power constraints.

The rest of the paper is organized as follows. In Section II, we introduce the system model and define the achievable rate region. In Sections [II and IV] we characterize the achievable rate regions with two different assumptions on the channel reciprocity. Sub-optimal schemes with lower complexity are discussed in Section $\mathrm{V}$ Numerical results are presented in Section VI with conclusions in Section VII

Notations: We use uppercase bold letters to denote matrices and lowercase bold letters to denote vectors. The conjugate, transpose, and Hermitian transpose are denoted by $(\cdot)^{*},(\cdot)^{T}$, and $(\cdot)^{H}$, respectively. The phase of a complex variable $a$ is denoted as $\angle a$. We use $\operatorname{tr}(\cdot)$ and $\operatorname{rank}(\cdot)$ to represent the trace and the rank of a matrix, respectively. A diagonal matrix with the elements of vector a as diagonal entries is denoted as $\operatorname{diag}(\mathbf{a})$. $\mathbf{A} \succeq 0$ means $\mathbf{A}$ is positive semi-definite, $\mathbf{a} \succeq \mathbf{b}$ means $a_{i} \geq b_{i}$ component-wise, and $\odot$ stands for the Hadamard (elementwise) multiplication.

\section{SYSTEM MODEL}

As shown in Fig. 1, we consider a collaborative two-way relay system consisting of two source nodes S1 and $\mathrm{S} 2$, each with a single antenna, and a relay cluster with $K$ single-antenna relay nodes $R_{i}$ 's, $i=1, \cdots, K$. No direct links between S1 and S2 exist. The forward channels from S1 and S2 to relay node $i$ are denoted as $h_{1, i}$ and $h_{2, i}$, respectively, while $h_{1, i}^{r}$ and $h_{2, i}^{r}$ denote the backward channels from relay node $i$ to $\mathrm{S} 1$ and $\mathrm{S} 2$, respectively. All the involved channels are assumed to take complex values and remain constant during one operation period. In addition, all channel state information is revealed to S1, S2, and the design/control center where the beamforming solution is solved. 
The two-way relaying takes two consecutive equal-length time-slots to finish one round of communication between $\mathrm{S} 1$ and $\mathrm{S} 2$ via the relay cluster with perfect synchronization assumed among $\mathrm{S} 1, \mathrm{~S} 2$, and $R_{i}, i=1, \cdots, K$. In the first time-slot, S1 and S2 transmit their signals simultaneously to the relay cluster; the $i$-th relay node receives the mixed signal $t_{i}(n)$, which is expressed as

$$
t_{i}(n)=h_{1, i} s_{1}(n)+h_{2, i} s_{2}(n)+v_{i}(n),
$$

where $s_{1}(n)$ and $s_{2}(n)$ are the transmitted symbols at time index $n$; and $v_{i}(n)$ is the receiver noise at relay node $i$, which is assumed to be circularly symmetric complex Gaussian (CSCG) with zero mean and variance $\sigma_{i}^{2}$. In the second time-slot, upon receiving the mixed signal, relay node $i$ multiplies a complex coefficient $w_{i}$ and forwards the signal, which is given as $u_{i}(n)=w_{i} t_{i}(n)$. At the source node terminals, $\mathbf{S} 1$ and $\mathbf{S} 2$ receive the sum signals from all the relay nodes, which are respectively given as

$$
\begin{aligned}
& y_{1}(n)=\sum_{i=1}^{K} h_{1, i}^{r} u_{i}(n)+z_{1}(n), \\
& y_{2}(n)=\sum_{i=1}^{K} h_{2, i}^{r} u_{i}(n)+z_{2}(n),
\end{aligned}
$$

where $z_{1}(n)$ and $z_{2}(n)$ are the noises at $\mathrm{S} 1$ and $\mathbf{S} 2$, respectively, which are assumed to be CSCG with zero mean and variances $\sigma_{S 1}^{2}$ and $\sigma_{S 2}^{2}$, respectively. Since S1 and S2 know their own transmitted signals, $s_{1}(n)$ and $s_{2}(n)$, respectively, they could subtract the resulting self-interference terms $\sum_{i=1}^{K} h_{1, i}^{r} w_{i} h_{1, i} s_{1}(n)$ and $\sum_{i=1}^{K} h_{2, i}^{r} w_{i} h_{2, i} s_{2}(n)$ from the received signals, respectively. Accordingly, the remaining signals for S1 and S2 are

$$
\begin{aligned}
& \tilde{y}_{1}(n)=\sum_{i=1}^{K}\left[h_{1, i}^{r} w_{i} h_{2, i} s_{2}(n)+h_{1, i}^{r} w_{i} v_{i}(n)\right]+z_{1}(n), \\
& \tilde{y}_{2}(n)=\sum_{i=1}^{K}\left[h_{2, i}^{r} w_{i} h_{1, i} s_{1}(n)+h_{2, i}^{r} w_{i} v_{i}(n)\right]+z_{2}(n) .
\end{aligned}
$$

Therefore, for a given $\mathbf{w}=\left[w_{1}, \cdots, w_{K}\right]^{T}$ the maximum achievable rates for the end-to-end link from $\mathbf{S} 2$ to $\mathrm{S} 1$ and from $\mathrm{S} 1$ to $\mathrm{S} 2$ are respectively given as

$$
\begin{aligned}
& R_{1}=\frac{1}{2} \log _{2}\left(1+\frac{P_{S 2}\left|\mathbf{f}_{2}^{T} \mathbf{w}\right|^{2}}{\sigma_{S 1}^{2}+\mathbf{w}^{H} \mathbf{A}_{1} \mathbf{w}}\right) \\
& R_{2}=\frac{1}{2} \log _{2}\left(1+\frac{P_{S 1}\left|\mathbf{f}_{1}^{T} \mathbf{w}\right|^{2}}{\sigma_{S 2}^{2}+\mathbf{w}^{H} \mathbf{A}_{2} \mathbf{w}}\right)
\end{aligned}
$$

where $\mathbf{f}_{1}=\mathbf{h}_{1} \odot \mathbf{h}_{2}^{r}, \mathbf{f}_{2}=\mathbf{h}_{2} \odot \mathbf{h}_{1}^{r}$ with $\mathbf{h}_{i}=\left[h_{i, 1}, \cdots, h_{i, K}\right]^{T}$ and $\mathbf{h}_{i}^{r}=\left[h_{i, 1}^{r}, \cdots, h_{i, K}^{r}\right]^{T}, i=1,2$. In addition, $\mathbf{A}_{1}=$ $\operatorname{diag}\left[\left|h_{1,1}^{r}\right|^{2} \sigma_{1}^{2}, \cdots,\left|h_{1, K}^{r}\right|^{2} \sigma_{K}^{2}\right], \mathbf{A}_{2}=\operatorname{diag}\left[\left|h_{2,1}^{r}\right|^{2} \sigma_{1}^{2}, \cdots,\left|h_{2, K}^{r}\right|^{2} \sigma_{K}^{2}\right], P_{S 1}$ and $P_{S 2}$ are the maximum transmit powers at $\mathrm{S} 1$ and $\mathrm{S} 2$, respectively, and the factor $1 / 2$ is due to the use of two orthogonal time-slots for relaying. 
Accordingly, we can define the set of rate pairs achievable by all feasible beamforming vector w's as

$$
\mathcal{R}=\bigcup_{\mathbf{w} \in \Omega_{w}}\left\{\left(r_{1}, r_{2}\right): r_{1} \leq R_{1}, r_{2} \leq R_{2}\right\}
$$

where the feasible set $\Omega_{w}$ can be defined by either a sum-power constraint or individual-power constraints. Specifically, when the sum-power constraint is considered, we have $\Omega_{w}=\left\{\mathbf{w}: p_{R}(\mathbf{w}) \leq P_{R}\right\}$, where $P_{R}$ is a scalar power limit and $p_{R}(\mathbf{w})$ is the sum-power of the relay cluster given the beamforming vector $\mathbf{w}$. When individual-power constraints are considered, we have $\Omega_{w}=\left\{\mathbf{w}: \mathbf{p}_{R}(\mathbf{w}) \preceq \mathbf{P}_{R}\right\}$, where $\mathbf{p}_{R}(\mathbf{w})$ is a vector of individual transmit powers, $\mathbf{P}_{R}$ is a vector with its elements denoting the power constraints for individual relay nodes, and $\preceq$ is element-wise.

When time-sharing between different achievable rate pairs is considered, the achievable rate region is then defined as the convex hull over the set of $\mathcal{R}$.

Definition 1: The achievable rate region $\mathcal{O}$ is the convex hull over the set of achievable rate pairs $\mathcal{R}$, i.e.,

$$
\mathcal{O}=H_{\mathrm{cvx}}(\mathcal{R})
$$

where $H_{\mathrm{cvx}}(\cdot)$ is the convex hull operation.

The goal of this paper is to efficiently characterize the achievable rate region $\mathcal{O}$. According to different assumptions on the channel reciprocity between the forward and backward channels, we first study the reciprocal case, and then study the non-reciprocal case.

\section{Reciprocal Channel CASE}

In this section, we assume that the forward channels from each source node to the relay nodes are reciprocal to the backward channels from the relay nodes to each corresponding source node, i.e., $h_{1, i}=h_{1, i}^{r}$ and $h_{2, i}=h_{2, i}^{r}$, for $i=1, \cdots, K$, which usually holds for a time-division-duplex (TDD) relaying system. In this case, it is obvious that when $\angle w_{i}=-\left(\angle h_{1, i}+\angle h_{2, i}\right)$, for $i=1, \cdots, K$, both rates given by (6) and (7) are maximized for a given set of $\left|w_{i}\right|$ 's. Thus, we only need to further find the optimal amplitudes for the elements in w. Let $x_{i}=\left|w_{i}\right|$ and $\hat{f}_{i}=\left|h_{1, i}\right|\left|h_{2, i}\right|$; we rewrite (6) and (7), respectively, as

$$
\begin{aligned}
& R_{1}=\frac{1}{2} \log _{2}\left(1+\frac{P_{S 2}\left(\hat{\mathbf{f}}^{T} \mathbf{x}\right)^{2}}{\sigma_{S 1}^{2}+\mathbf{x}^{T} \mathbf{A}_{1} \mathbf{x}}\right), \\
& R_{2}=\frac{1}{2} \log _{2}\left(1+\frac{P_{S 1}\left(\hat{\mathbf{f}}^{T} \mathbf{x}\right)^{2}}{\sigma_{S 2}^{2}+\mathbf{x}^{T} \mathbf{A}_{2} \mathbf{x}}\right),
\end{aligned}
$$

where $\mathbf{x}=\left[\left|w_{1}\right|, \cdots,\left|w_{K}\right|\right]^{T}$ and $\hat{\mathbf{f}}=\left[\left|h_{1,1}\right|\left|h_{2,1}\right|, \cdots,\left|h_{1, K}\right|\left|h_{2, K}\right|\right]^{T}$. In order to obtain $\mathcal{O}$, we need to characterize the Pareto boundary of $\mathcal{R}$. A common method is via solving a sequence of weighted sum-rate maximization 
(WSRMax) problems [15], each for a different non-negative weight vector $(\lambda, 1-\lambda), 0 \leq \lambda \leq 1$, as follows

$$
\begin{array}{ll}
\max _{\mathbf{x}} & \frac{\lambda}{2} \log _{2}\left(1+\frac{P_{S 2}\left(\hat{\mathbf{f}}^{T} \mathbf{x}\right)^{2}}{\sigma_{S 1}^{2}+\mathbf{x}^{T} \mathbf{A}_{1} \mathbf{x}}\right) \\
& +\frac{1-\lambda}{2} \log _{2}\left(1+\frac{P_{S 1}\left(\hat{\mathbf{f}}^{T} \mathbf{x}\right)^{2}}{\sigma_{S 2}^{2}+\mathbf{x}^{T} \mathbf{A}_{2} \mathbf{x}}\right) \\
\text { s.t. } & \mathbf{x} \in \Omega_{w} .
\end{array}
$$

Unfortunately, we cannot derive the closed-form solution for the WSRMax problem. However, from (10) and (11), we see that the received SNRs at S1 and S2 are

$$
\begin{aligned}
\mathrm{SNR}_{1} & =\frac{P_{S 2}\left(\hat{\mathbf{f}}^{T} \mathbf{x}\right)^{2}}{\sigma_{S 1}^{2}+\mathbf{x}^{T} \mathbf{A}_{1} \mathbf{x}}, \\
\mathrm{SNR}_{2} & =\frac{P_{S 1}\left(\hat{\mathbf{f}}^{T} \mathbf{x}\right)^{2}}{\sigma_{S 2}^{2}+\mathbf{x}^{T} \mathbf{A}_{2} \mathbf{x}},
\end{aligned}
$$

respectively, where their numerators differ by only a scalar constant. As shown later, for each given weight vector $(\mu, 1-\mu), 0 \leq \mu \leq 1$, we could easily find a closed-form solution for the following weighted sum inverse-SNRs minimization(WSISMin) problem

$$
\begin{array}{ll}
\min _{\mathbf{x}} & \mu \frac{\sigma_{S 1}^{2}+\mathbf{x}^{T} \mathbf{A}_{1} \mathbf{x}}{P_{S 2}\left(\hat{\mathbf{f}}^{T} \mathbf{x}\right)^{2}}+(1-\mu) \frac{\sigma_{S 2}^{2}+\mathbf{x}^{T} \mathbf{A}_{2} \mathbf{x}}{P_{S 1}\left(\hat{\mathbf{f}}^{T} \mathbf{x}\right)^{2}} \\
\text { s.t. } & \mathbf{x} \in \Omega_{w} .
\end{array}
$$

Hence, we could quantify the Pareto boundary for the inverse-SNR region. Based on this observation, together with the fact that there exists a bijective mapping between an inverse-SNR pair and a rate pair, we are inspired to probe the question on whether we could construct the achievable rate region $\mathcal{O}$ from the easily obtainable inverse-SNR region 1 . In the following, we first introduce some definitions related to the inverse-SNR region and then show that we indeed can construct the achievable rate region $\mathcal{O}$ from the inverse-SNR region, based on the set of closed-form solutions for a sequence of the WSISMin problems.

\section{A. Characterizing the Achievable Rate Region}

At first, we introduce some definitions.

Definition 2: Consider a bijective mapping $\mathcal{U}:(x, y) \mapsto\left(\frac{1}{2} \log _{2}(1+1 / x), \frac{1}{2} \log _{2}(1+1 / y)\right)$ with $(x, y) \in R_{++}^{2}$;

${ }^{1}$ In an independent work of [16], the authors also applied the approach of inverse-SNR. However, their motivation and objective are completely different from ours, where they use the sum-MSE as the objective and use the approximation $M S E \approx 1 / S N R$ to transform the sum-MSE into the sum of inverse-SNR. Hence, their results only hold for high SNR cases. In addition, they apply the beamformer that corresponds to the minimum sum-MSE directly to maximize the sum-rate. The optimality is suspicious since the beamformer that minimizes the sum-MSE is not necessary the beamformer that maximizes the sum-rate. 
then the set of achievable inverse-SNR pairs $\mathcal{I}$ is defined as

$$
\mathcal{I}=\left\{\left(t_{1}, t_{2}\right): \mathcal{U}\left(t_{1}, t_{2}\right) \in \mathcal{R}\right\}
$$

For regions $\mathcal{R}$ and $\mathcal{I}$, we are particularly interested in their Pareto boundaries, which are defined as follows.

Definition 3: The Pareto boundary of $\mathcal{R}$ is defined as $\mathcal{P}=\left\{\left(r_{1}, r_{2}\right):\left(r_{1}, r_{2}\right) \in \mathcal{R},\left[\left(r_{1}, r_{2}\right)+\mathbf{K}\right] \cap \mathcal{R}=\right.$ $\left.\left(r_{1}, r_{2}\right)\right\}$, and the Pareto boundary of $\mathcal{I}$ is defined as $\mathcal{B}=\left\{\left(t_{1}, t_{2}\right):\left(t_{1}, t_{2}\right) \in \mathcal{I},\left[\left(t_{1}, t_{2}\right)-\mathbf{K}\right] \cap \mathcal{I}=\left(t_{1}, t_{2}\right)\right\}$, where $\mathbf{K}=\mathbf{R}_{+}^{2}$ is a non-negative cone [15].

Definition 4: Define the points obtained by solving the WSISMin problem with a given weight vector $(\mu, \bar{\mu})$ as

$$
\mathcal{S}(\mu, \mathcal{I})=\left\{\left(t_{1}, t_{2}\right): \min _{\left(t_{1}, t_{2}\right) \in \mathcal{I}} \mu t_{1}+\bar{\mu} t_{2}\right\}
$$

where $\bar{\mu}=1-\mu$.

Definition 5: The set of points that can be obtained from a sequence of WSISMin problems is given as

$$
\mathcal{S}(\mathcal{I})=\bigcup_{0 \leq \mu \leq 1} \mathcal{S}(\mu, \mathcal{I})
$$

In order to show that we can construct $\mathcal{O}$ from $\mathcal{S}(\mathcal{I})$, where $\mathcal{O}$ can be obtained by convex hulling over $\mathcal{P}$, we need to prove two things: (1) A point in $\mathcal{B}$ could be mapped to a point in $\mathcal{P}$ and vice versa, which means $\mathcal{U}(\mathcal{B})=\mathcal{P}$; (2) The points in $\mathcal{B}$ that cannot be obtained by WSISMin are mapped to the points in $\mathcal{P}$ that are unnecessary for constructing $\mathcal{O}$ by convex hulling over $\mathcal{P}$, i.e., $\mathcal{U}(\mathcal{B} \backslash \mathcal{S}(\mathcal{I})) \subseteq \mathcal{P}_{\text {non }}$, where $\mathcal{P}_{\text {non }}$ denotes the set of points in $\mathcal{P}$ that are unnecessary for constructing $\mathcal{O}$ by convex hulling over $\mathcal{P}$. With the above two statements hold, it is easy to see that $\mathcal{P} \backslash \mathcal{P}_{\text {non }} \subseteq \mathcal{U}(\mathcal{S}(\mathcal{I})$ ), i.e., the points obtained by WSISMin suffice to construct $\mathcal{O}$.

Proposition 1: The Pareto boundary of the inverse-SNR region can be mapped to the Pareto boundary of the rate region $\mathcal{R}$ by mapping $\mathcal{U}$ as given in Definition 2 and vice versa, i.e.,

$$
\mathcal{P}=\mathcal{U}(\mathcal{B})
$$

Proof: See Appendix in VIII-A

Proposition 2: The image of $\mathcal{B} \backslash \mathcal{S}(\mathcal{I})$ is not necessary for constructing the achievable rate region $\mathcal{O}$.

Proof: See Appendix in VIII-B.

Theorem 1: The points in $\mathcal{S}(\mathcal{I})$ are sufficient to construct the achievable rate region $\mathcal{O}$.

Proof: Since $\mathcal{P}=\mathcal{U}(\mathcal{B})$ and $\mathcal{U}(\mathcal{B} \backslash \mathcal{S}(\mathcal{I}))$ in $\mathcal{P}$ is not necessary for constructing the achievable rate region $\mathcal{O}$, it is easy to see that $\mathcal{U}(\mathcal{S}(\mathcal{I})$ ) suffices to construct the achievable rate region $\mathcal{O}$ given that $\mathcal{O}$ is obtained by convex hulling over $\mathcal{P}$. 
Since we have shown that $\mathcal{S}(\mathcal{I})$ suffices to construct the achievable rate region $\mathcal{O}$, instead of studying the problem in (12) and (13), we now study the solutions of the WSISMin problems in the following.

\section{B. Collaborative Beamforming under Sum-power Constraint}

In this subsection, we consider the case where the relay cluster has a sum-power constraint. The total transmit power of the relay cluster is

$$
\begin{aligned}
p_{R} & =\sum_{i=1}^{K}\left(\left|x_{i} h_{1, i}\right|^{2} P_{S 1}+\left|x_{i} h_{2, i}\right|^{2} P_{S 2}+\left|x_{i}\right|^{2} \sigma_{i}^{2}\right) \\
& =\mathbf{x}^{H} \mathbf{D} \mathbf{x}
\end{aligned}
$$

where we have $\mathbf{D}=\operatorname{diag}\left[\left|h_{1,1}\right|^{2} P_{S 1}+\left|h_{2,1}\right|^{2} P_{S 2}+\sigma_{1}^{2}, \cdots,\left|h_{1, K}\right|^{2} P_{S 1}+\left|h_{2, K}\right|^{2} P_{S 2}+\sigma_{K}^{2}\right]$. According to the discussion in the last subsection, to quantify the rate region is equivalent to seeking the optimal solutions for the WSISMin problems given the sum-power constraint as follows:

$$
\begin{array}{ll}
\min _{\mathbf{x}} & \mu / \mathrm{SNR}_{1}+\bar{\mu} / \mathrm{SNR}_{2} \\
\text { s.t. } & \mathbf{x}^{T} \mathbf{D} \mathbf{x} \leq P_{R},
\end{array}
$$

where $0<\mu<1$ is the weight. First of all, the optimal $\mathbf{x}^{*}$ must satisfy $\mathbf{x}^{* T} \mathbf{D} \mathbf{x}^{*}=P_{R}$; otherwise, we can always scale up $\mathrm{x}$ such that the objective function is decreased. When $\mu=0$ or 1 , this problem degrades to find the optimal beamforming vector for collaborative one-way relay, which has been extensively studied in [5], [7], and [16].

Given the SNRs from (14) and (15) and the fact of $\mathbf{x}^{* T} \mathbf{D} \mathbf{x}^{*}=P_{R}$, the optimal $\mathbf{x}$ for (23) should be the solution of the following problem:

$$
\min _{\mathbf{x}} \frac{\mathbf{x}^{T}\left[\nu \mathbf{D} / P_{R}+\mu / P_{S 2} \mathbf{A}_{1}+\bar{\mu} / P_{S 1} \mathbf{A}_{2}\right] \mathbf{x}}{\mathbf{x}^{T} \hat{\mathbf{f}} \hat{\mathbf{f}}^{T} \mathbf{x}}
$$

where $\nu=\mu \sigma_{S 1}^{2} / P_{S 2}+\bar{\mu} \sigma_{S 2}^{2} / P_{S 1}$. The above problem is equivalent to

$$
\max _{\mathbf{x}} \frac{\mathbf{x}^{T} \hat{\mathbf{f}} \hat{\mathbf{f}}^{T} \mathbf{x}}{\mathbf{x}^{T}\left[\nu \mathbf{D} / P_{R}+\mu / P_{S 2} \mathbf{A}_{1}+\bar{\mu} / P_{S 1} \mathbf{A}_{2}\right] \mathbf{x}}
$$

which can be written in the form of Rayleigh-Ritz ratio [17] and the optimal solution is thus given as

$$
\mathbf{x}^{*}=\xi \boldsymbol{\Gamma}^{-1} \hat{\mathbf{f}} /\left\|\boldsymbol{\Gamma}^{-1} \hat{\mathbf{f}}\right\|,
$$




$$
\begin{aligned}
& \mathrm{SNR}_{1}= \frac{P_{S 2}\left(\sum_{i=1}^{K}\left|h_{1, i}\right|\left|h_{2, i}\right| \sqrt{\frac{p_{i}}{\sigma_{i}^{2}+P_{S 1}\left|h_{1, i}\right|^{2}+P_{S 2}\left|h_{2, i}\right|^{2}}} \alpha_{i}\right)^{2}}{\sigma_{S 1}^{2}+\sum_{i=1}^{K} \frac{\sigma_{i}^{2}\left|h_{1, i}\right|_{i}^{2} \alpha_{i}^{2} p_{i}}{\sigma_{i}^{2}+P_{S 1}\left|h_{1, i}\right|^{2}+P_{S 2}\left|h_{2, i}\right|^{2}}} \\
& \mathrm{SNR}_{2}=\frac{P_{S 1}\left(\sum_{i=1}^{K}\left|h_{2, i}\right|\left|h_{1, i}\right| \sqrt{\frac{p_{i}}{\sigma_{i}^{2}+P_{S 1}\left|h_{1, i}\right|^{2}+P_{S 2}\left|h_{2, i}\right|^{2}}} \alpha_{i}\right)^{2}}{\sigma_{S 2}^{2}+\sum_{i=1}^{K} \frac{\sigma_{i}^{2}\left|h_{2, i}\right|^{2} \alpha_{i}^{2} p_{i}}{\sigma_{i}^{2}+P_{S 1}\left|h_{1, i}\right|^{2}+P_{S 2}\left|h_{2, i}\right|^{2}}}
\end{aligned}
$$

where

$$
\begin{aligned}
\boldsymbol{\Gamma} & =\operatorname{diag}\left[\nu \frac{\beta_{1}}{P_{R}}+\eta_{1}, \cdots, \nu \frac{\beta_{K}}{P_{R}}+\eta_{K}\right], \\
\beta_{i} & =\sigma_{i}^{2}+P_{S 1}\left|h_{1, i}\right|^{2}+P_{S 2}\left|h_{2, i}\right|^{2}, \\
\eta_{i} & =\sigma_{i}^{2}\left(\left|h_{1, i}\right|^{2} \mu / P_{S 1}+\left|h_{2, i}\right|^{2} \bar{\mu} / P_{S 2}\right),
\end{aligned}
$$

and $\xi$ is a scalar such that $\mathbf{x}^{* T} \mathbf{D} \mathbf{x}^{*}=P_{R}$. By searching over all $\mu$ 's, we derived a set of $\mathbf{x}^{*}$ 's and hence we could compute a set of rate pairs by injecting (27) into (10) and (11). The achievable rate region $\mathcal{O}$ is then obtained by convex-hulling over such a set of rate pairs.

Partially distributed implementation: After exhausting all $\mu$ 's, we set up a lookup table for rate-pairs indexed by $\mu$. During normal operations, the control center first looks up the table to decides the appropriate $\mu$ such that S1 and S2 achieve a desirable rate pair; and it broadcasts $\mu$ and the global constant $\xi /\left\|\boldsymbol{\Gamma}^{-1} \hat{\mathbf{f}}\right\|$, while $P_{S 1}, P_{S 2}$, $\sigma_{S 1}, \sigma_{S 2}$ are constant and assumed to be known at all the relays. Upon receiving the broadcast message from the control center, each relay node determines the optimal $w_{i}$ from its local information $h_{1, i}$ and $h_{2, i}$, which is given as

$$
w_{i}=\frac{\xi}{\left\|\boldsymbol{\Gamma}^{-1} \hat{\mathbf{f}}\right\|} \frac{\left|h_{1, i}\right|\left|h_{2, i}\right|}{\nu \beta_{i} / P_{R}+\eta_{i}} e^{-j\left(\angle h_{1, i}+\angle h_{2, i}\right)}
$$

\section{Collaborative Beamforming under Individual-Power Constraints}

In the previous subsection, we assume that the relay cluster has a sum-power constraint. In practice, each relay may have its own power constraint due to the individual power supplies. The transmit power at relay $i$ is given as

$$
p_{R, i}=\left|x_{i}\right|^{2}\left(\left|h_{1, i}\right|^{2} P_{S 1}+\left|h_{2, i}\right|^{2} P_{S 2}+\sigma_{i}^{2}\right),
$$

where $p_{R, i} \leq p_{i}$, with $p_{i}$ is the maximum allowable power for relay node $i$. Equivalently, we could set $p_{R, i}=\alpha_{i}^{2} p_{i}$ with $0 \leq \alpha_{i} \leq 1$ as a new design variable. Correspondingly, the received SNRs can be rewritten as (33) and (34) 
Let

$$
\begin{aligned}
\mathbf{H}_{1} & =\operatorname{diag}\left[\sigma_{1}^{2} p_{1}\left|h_{1,1}\right|^{2}, \cdots, \sigma_{K}^{2} p_{K}\left|h_{1, K}\right|^{2}\right], \\
\mathbf{H}_{2} & =\operatorname{diag}\left[\sigma_{1}^{2} p_{1}\left|h_{2,1}\right|^{2}, \cdots, \sigma_{K}^{2} p_{K}\left|h_{2, K}\right|^{2}\right], \\
g_{i} & =\sqrt{p_{i}}\left|h_{1, i}\right|\left|h_{2, i}\right| / \sqrt{\mathbf{D}_{i, i}} .
\end{aligned}
$$

We can recast (33) and (34) as

$$
\begin{aligned}
\mathrm{SNR}_{1} & =\frac{P_{S 2} \boldsymbol{\alpha}^{\boldsymbol{T}} \mathbf{g g}^{T} \boldsymbol{\alpha}}{\sigma_{S 1}^{2}+\boldsymbol{\alpha}^{\boldsymbol{T}} \mathbf{H}_{1} \mathbf{D}^{-1} \boldsymbol{\alpha}} \\
\mathrm{SNR}_{2} & =\frac{P_{S 1} \boldsymbol{\alpha}^{\boldsymbol{T}} \mathbf{g g}^{T} \boldsymbol{\alpha}}{\sigma_{S 2}^{2}+\boldsymbol{\alpha}^{\boldsymbol{T}} \mathbf{H}_{2} \mathbf{D}^{-1} \boldsymbol{\alpha}}
\end{aligned}
$$

respectively, where $\mathbf{0} \preceq \boldsymbol{\alpha} \preceq \mathbf{1}$. The WSISMin problem for the individual-power constraint case is now given as:

$$
\min _{\mathbf{0} \preceq \boldsymbol{\alpha} \preceq \mathbf{1}} \frac{\nu+\boldsymbol{\alpha}^{T}\left(\mathbf{H}_{1} \mathbf{D}^{-1} \mu / P_{S 2}+\mathbf{H}_{2} \mathbf{D}^{-1} \bar{\mu} / P_{S 1}\right) \boldsymbol{\alpha}}{\boldsymbol{\alpha}^{T} \mathbf{g g}^{T} \boldsymbol{\alpha}},
$$

which is equivalent to solve

$$
\max _{\mathbf{0} \preceq \boldsymbol{\alpha} \preceq \mathbf{1}} \frac{\boldsymbol{\alpha}^{T} \mathbf{g g}^{T} \boldsymbol{\alpha}}{\nu+\boldsymbol{\alpha}^{T}\left(\mathbf{H}_{1} \mathbf{D}^{-1} \mu / P_{S 2}+\mathbf{H}_{2} \mathbf{D}^{-1} \bar{\mu} / P_{S 1}\right) \boldsymbol{\alpha}} .
$$

For notation simplicity, let

$$
\begin{aligned}
\boldsymbol{\Psi} & =\left[\left(\mathbf{H}_{1} \mu / P_{S 2}+\mathbf{H}_{2} \bar{\mu} / P_{S 1}\right) \mathbf{D}^{-1} / \nu\right]^{1 / 2} \\
\tilde{\mathbf{g}} & =\mathbf{g} / \sqrt{\nu}
\end{aligned}
$$

where $\Psi$ is diagonal with its diagonal elements denoted as $\psi_{i}, i=1, \cdots, K$. Then the above problem becomes

$$
\max _{\mathbf{0} \preceq \boldsymbol{\alpha} \preceq \mathbf{1}} \frac{\langle\tilde{\mathbf{g}}, \boldsymbol{\alpha}\rangle^{2}}{1+\|\boldsymbol{\Psi} \boldsymbol{\alpha}\|^{2}} .
$$

For each given $\mu$, (44] can be solved analytically by following the results in [8]. Before we present the solution, we first define $\phi_{i}=\tilde{g}_{i} / \psi_{i}^{2}$ for $i=1, \cdots, K$ and $\phi_{K+1}=0$. Then we sort $\phi_{i}$ as $\phi_{\tau_{1}} \geq \phi_{\tau_{2}} \geq \cdots \geq \phi_{\tau_{K}} \geq \phi_{\tau_{K+1}}$. Moreover, let $\lambda_{k}=\frac{1+\sum_{m=1}^{k} \psi_{\tau_{m}}^{2}}{\sum_{m=1}^{k} \tilde{g}_{\tau_{m}}}$ and define the $j$-th element of the vector $\boldsymbol{\alpha}^{(k)}$ as

$$
\alpha_{j}^{(k)}=\left\{\begin{array}{c}
1, \quad j=\tau_{1}, \cdots \tau_{k} \\
\lambda_{k} \phi_{j}, \quad j=\tau_{k+1}, \cdots \tau_{K}
\end{array}\right.
$$

Then the solution for (44) is given by following theorem.

Theorem 2: The solution of (44) is $\boldsymbol{\alpha}^{\left(k^{*}\right)}$ given by [45), where $k^{*}$ is the smallest $k$ such that $\lambda_{k}<\phi_{\tau_{k+1}}^{-1}$.

Proof: This result directly follows the results in [8]. 
Partially distributed implementation: Besides the value of $\mu$, the control center only needs to broadcast $\lambda_{k^{*}}$ at each operation period. Each relay node then determines $\phi_{i}$ with its local information. If $\phi_{i}^{-1} \leq \lambda_{k^{*}}$, the relay node transmits at its maximum power. Otherwise, it transmits with power $\left(\lambda_{k^{*}} \phi_{i}\right)^{2} p_{i}$, i.e., the optimal $w_{i}=$

$\alpha_{i}^{\left(k^{*}\right)} \sqrt{p_{i}} e^{-j\left(\angle h_{1, i}+\angle h_{2, i}\right)}$, where $\alpha_{i}^{\left(k^{*}\right)}$ is given in (45). From the solutions, we see that in general some relay nodes may not transmit with maximum transmit power.

\section{Non-Reciprocal Channel CASE}

In the last section, we have discussed the case where the uplink and downlink channels are reciprocal. In this section, we discuss the case where the uplink and downlink channels are non-reciprocal, which may be the result of deploying frequency-division-duplex (FDD) system.

Due to the lack of channel reciprocity, the approach taken in the last section does not apply here. In order to characterize the boundary of the region $\mathcal{R}$, as we discussed before a commonly used method is to solve the following

$$
\begin{aligned}
\max _{\mathbf{w}} & \frac{\lambda}{2} \log _{2}\left(1+\frac{P_{S 2}\left|\mathbf{f}_{2}^{T} \mathbf{w}\right|^{2}}{\sigma_{S 1}^{2}+\mathbf{w}^{H} \mathbf{A}_{1} \mathbf{w}}\right) \\
& +\frac{1-\lambda}{2} \log _{2}\left(1+\frac{P_{S 1}\left|\mathbf{f}_{1}^{T} \mathbf{w}\right|^{2}}{\sigma_{S 2}^{2}+\mathbf{w}^{H} \mathbf{A}_{2} \mathbf{w}}\right) \\
\text { s.t. } & \mathbf{w} \in \Omega_{w},
\end{aligned}
$$

for each given weight vector $(\lambda, 1-\lambda)$. However, the above problem is non-convex since the objective function is not a concave function. To efficiently quantify the rate region, here we resort to an alternative method called the rate-profile method [9], formulated as

$$
\begin{aligned}
\max _{\mathbf{w}, R_{\text {sum }}} & R_{\text {sum }} \\
\text { s.t. } & \frac{1}{2} \log _{2}\left(1+\frac{P_{S 2}\left|\mathbf{f}_{2}^{T} \mathbf{w}\right|^{2}}{\sigma_{S 1}^{2}+\mathbf{w}^{H} \mathbf{A}_{1} \mathbf{w}}\right) \geq \kappa R_{\text {sum }}, \\
& \frac{1}{2} \log _{2}\left(1+\frac{P_{S 1}\left|\mathbf{f}_{1}^{T} \mathbf{w}\right|^{2}}{\sigma_{S 2}^{2}+\mathbf{w}^{H} \mathbf{A}_{2} \mathbf{w}}\right) \geq \bar{\kappa} R_{\text {sum }}, \\
& \mathbf{w} \in \Omega_{w},
\end{aligned}
$$

where $R_{\text {sum }}$ is the sum rate given a rate profile vector $[\kappa, \bar{\kappa}]$ with $0 \leq \kappa \leq 1$ and $\bar{\kappa}=1-\kappa$. Let $\mathbf{F}_{1}=\mathbf{f}_{1}^{*} \mathbf{f}_{1}^{T}$, 
$\mathbf{F}_{2}=\mathbf{f}_{2}^{*} \mathbf{f}_{2}^{T}$, and $\mathbf{X}=\mathbf{w w}^{H}$. The above problem is equivalent to

$$
\begin{aligned}
\max _{\mathbf{X}, R_{\text {sum }}} & R_{\text {sum }} \\
\text { s.t. } & \frac{1}{2} \log _{2}\left(1+\frac{P_{S 2} \operatorname{tr}\left(\mathbf{F}_{2} \mathbf{X}\right)}{\sigma_{S 1}^{2}+\operatorname{tr}\left(\mathbf{A}_{\mathbf{1}} \mathbf{X}\right)}\right) \geq \kappa R_{\text {sum }}, \\
& \frac{1}{2} \log _{2}\left(1+\frac{P_{S 1} \operatorname{tr}\left(\mathbf{F}_{1} \mathbf{X}\right)}{\sigma_{S 2}^{2}+\operatorname{tr}\left(\mathbf{A}_{\mathbf{2}} \mathbf{X}\right)}\right) \geq \bar{\kappa} R_{\text {sum }}, \\
& \mathbf{X} \in \Omega_{X}, \\
& \mathbf{X} \succeq 0, \\
& \operatorname{rank}(\mathbf{X})=1,
\end{aligned}
$$

where the last constraint $\operatorname{rank}(\mathbf{X})=1$ comes from the fact $\mathbf{X}=\mathbf{w w}^{H}$, and $\Omega_{X}=\left\{\mathbf{X}: \mathbf{X}=\mathbf{w w}^{H}, \mathbf{w} \in \Omega_{w}\right\}$ and $\Omega_{w}$ is defined after (8). According to different assumptions on the power constraint, the above problem can be further converted into different semi-definite programming (SDP) problems after semi-definite relaxation (SDR).

\section{A. Sum-power Constrained Case}

In this subsection, we assume that the relay cluster operates under a sum-power constraint $P_{R}$. Given the sumpower constraint, the power constraint in (55) can be replaced by $\operatorname{tr}(\mathbf{D X}) \leq P_{R}$, where $\mathbf{D}=\operatorname{diag}\left[\left|h_{1,1}\right|^{2} P_{S 1}+\right.$ $\left.\left|h_{2,1}\right|^{2} P_{S 2}+\sigma_{1}^{2}, \cdots,\left|h_{1, K}\right|^{2} P_{S 1}+\left|h_{2, K}\right|^{2} P_{S 2}+\sigma_{K}^{2}\right]$. Since the rank-one constraint is not convex, the problem is still not a convex problem and hence may not be efficiently solvable. To address this issue, let us first remove the rank-one constraint and consider the following relay power minimization problem for given set of $\kappa$ and $R_{\text {sum }}=r$ :

$$
\begin{array}{ll}
\min _{\mathbf{X}} & \operatorname{tr}(\mathbf{D X}) \\
\text { s.t. } & \frac{1}{2} \log _{2}\left(1+\frac{P_{S 2} \operatorname{tr}\left(\mathbf{F}_{2} \mathbf{X}\right)}{\sigma_{S 1}^{2}+\operatorname{tr}\left(\mathbf{A}_{1} \mathbf{X}\right)}\right) \geq \kappa r, \\
& \frac{1}{2} \log _{2}\left(1+\frac{P_{S 1} \operatorname{tr}\left(\mathbf{F}_{1} \mathbf{X}\right)}{\sigma_{S 2}^{2}+\operatorname{tr}\left(\mathbf{A}_{\mathbf{2}} \mathbf{X}\right)}\right) \geq \bar{\kappa} r, \\
& \mathbf{X} \succeq 0,
\end{array}
$$

which is equivalent to

$$
\begin{array}{ll}
\min _{\mathbf{X}} & \operatorname{tr}(\mathbf{D X}) \\
\text { s.t. } & \frac{P_{S 2} \operatorname{tr}\left(\mathbf{F}_{2} \mathbf{X}\right)}{\sigma_{S 1}^{2}+\operatorname{tr}\left(\mathbf{A}_{\mathbf{1}} \mathbf{X}\right)} \geq \gamma_{1}, \\
& \frac{P_{S 1} \operatorname{tr}\left(\mathbf{F}_{1} \mathbf{X}\right)}{\sigma_{S 2}^{2}+\operatorname{tr}\left(\mathbf{A}_{\mathbf{2}} \mathbf{X}\right)} \geq \gamma_{2}, \\
& \mathbf{X} \succeq 0,
\end{array}
$$


where $\gamma_{1}=2^{2 \kappa r}-1, \gamma_{2}=2^{2 \bar{\kappa} r}-1$, and they can be considered as the SNR constraints for S1 and S2, respectively. Since $\sigma_{S 1}^{2}+\operatorname{tr}\left(\mathbf{A}_{1} \mathbf{X}\right) \geq 0$ and $\sigma_{S 2}^{2}+\operatorname{tr}\left(\mathbf{A}_{2} \mathbf{X}\right) \geq 0$, we could rewrite the above problem as following SDP problem:

$$
\begin{array}{ll}
\min _{\mathbf{X}} & \operatorname{tr}(\mathbf{D X}) \\
\text { s.t. } & \operatorname{tr}\left[\left(P_{S 2} \mathbf{F}_{2}-\gamma_{1} \mathbf{A}_{1}\right) \mathbf{X}\right] \geq \gamma_{1} \sigma_{S 1}^{2}, \\
& \operatorname{tr}\left[\left(P_{S 1} \mathbf{F}_{1}-\gamma_{2} \mathbf{A}_{2}\right) \mathbf{X}\right] \geq \gamma_{2} \sigma_{S 2}^{2}, \\
& \mathbf{X} \succeq 0 .
\end{array}
$$

Denote the optimal value of the above problem as $p_{R}^{*}$, which is the minimum sum-power required by the relay cluster to support the target SNRs $\gamma_{1}$ and $\gamma_{2}$ for S1 and S2, respectively. If $p_{R}^{*} \leq P_{R}$, then $\left(\gamma_{1}, \gamma_{2}\right)$ must be an achievable SNR pair. Otherwise, $\gamma_{1}$ and $\gamma_{2}$ are not achievable. Based on this observation, we propose the following bi-section algorithm such that the problem (52) without rank-one constraint can be solved by solving a sequence of convex power feasibility problems, with the assumption that we know an upper bound for $R_{\text {sum }}$, denoted as $r_{\max }$. Algorithm 1:

- Initialize $r_{l o w}=0, r_{u p}=r_{\max }$.

- Repeat

1) Set $r \leftarrow \frac{1}{2}\left(r_{\text {low }}+r_{\text {up }}\right)$.

2) Solve problem (66)-696) with the given $r$.

3) Update $r$ with the bi-section method [15]: If $p_{R}^{*} \leq P_{R}$, set $r_{l o w}=r$; otherwise, $r_{u p}=r$.

- Until $r_{u p}-r_{\text {low }}<\epsilon$, where $\epsilon$ is a small positive accuracy parameter.

The rate upper bound $r_{\max }$ can be derived as follows. We first decouple the two-way relay channel into two one-way relay channels and obtain a rate for each one-way relay channel. Denote the larger rate as $\tilde{r}$. Then $r_{\max }$ can be set as $2 \tilde{r}$. The one-way collaborative relay beamforming with sum-power constraint is well-studied, and the rate can be derived from the results in [7].

1) Rank-one solution: The resulting optimal solution $\mathbf{X}_{\text {opt }}$ obtained from Algorithm 1 may not be of rank-one due to the SDP relaxation, which means that $\mathbf{X}_{o p t}$ may not lead to an optimal beamforming vector w. However, since there are only two linear constraints [67] and [68), it has been shown in [9] and [18] that an exact rank-one optimal solution can always be constructed from a non-rank-one optimal solution. The transformation techniques developed in [9] and [18] can be used to obtain the rank-one solution. Note that the beamforming solution for the non-reciprocal channel case is fully centralized, which cannot be implemented in a partially distributed fashion. 


\section{B. Individual-Power Constrained Case}

In the previous subsection, we have discussed the sum-power constrained case where the non-convex rate maximization problem is converted into a sequence of convex sum-power minimization problems. In this subsection, we put a stricter limitation on the relay power by assuming that each node has its individual power constraint. In this case, following a similar SDR technique to that in the previous subsection, the optimization problem with individual power constraints can be cast as

$$
\begin{aligned}
\max _{\mathbf{X}, R_{\text {sum }}} & R_{\text {sum }} \\
\text { s.t. } & \frac{P_{S 2} \operatorname{tr}\left(\mathbf{F}_{2} \mathbf{X}\right)}{\sigma_{S 1}^{2}+\operatorname{tr}\left(\mathbf{A}_{1} \mathbf{X}\right)} \geq \gamma_{1}, \\
& \frac{P_{S 1} \operatorname{tr}\left(\mathbf{F}_{1} \mathbf{X}\right)}{\sigma_{S 2}^{2}+\operatorname{tr}\left(\mathbf{A}_{2} \mathbf{X}\right)} \geq \gamma_{2}, \\
& \mathbf{D}_{i, i} \mathbf{X}_{i, i} \leq P_{R}^{i}, i=1, \cdots, K, \\
& \mathbf{X} \succeq 0,
\end{aligned}
$$

where $\mathbf{D}_{i, i}$ and $\mathbf{X}_{i, i}$ are the $i$-th diagonal elements of $\mathbf{D}$ and $\mathbf{X}$, respectively. The transmit power at node $i$ amounts to $\mathbf{D}_{i, i} \mathbf{X}_{i, i}$ and the individual power limit at node $i$ is $P_{R}^{i}$. However, we cannot translate the above problem into a sequence of power feasibility problems as given in the last subsection, since we now have $K$ individual power constraints rather than a single sum-power constraint for the whole relay cluster. Alternatively, we aim at solving a sequence of the following problem via bi-section search over $r$.

$$
\begin{array}{cl}
\max _{\mathbf{X}, r} & r \\
\text { s.t. } & \operatorname{tr}\left[\left(P_{S 2} \mathbf{F}_{2}-\gamma_{1} \mathbf{A}_{1}\right) \mathbf{X}\right] \geq \gamma_{1} \sigma_{S 1}^{2}, \\
& \operatorname{tr}\left[\left(P_{S 1} \mathbf{F}_{1}-\gamma_{2} \mathbf{A}_{2}\right) \mathbf{X}\right] \geq \gamma_{2} \sigma_{S 2}^{2}, \\
& \mathbf{X}(i, i) \leq P_{R}^{i} / \mathbf{D}(i, i), i=1, \cdots, K, \\
& \mathbf{X} \succeq 0 .
\end{array}
$$


The above problem is convex over $\mathbf{X}$ at each given value of $r$. Let $r^{*}$ be the maximum value obtained by solving (75). For a given value of $r$, we solve the following feasibility problem

$$
\begin{array}{ll}
\text { Find } & \mathbf{X} \\
\text { s.t. } & \operatorname{tr}\left[\left(P_{S 2} \mathbf{F}_{2}-\gamma_{1} \mathbf{A}_{1}\right) \mathbf{X}\right] \geq \gamma_{1} \sigma_{S 1}^{2}, \\
& \operatorname{tr}\left[\left(P_{S 1} \mathbf{F}_{1}-\gamma_{2} \mathbf{A}_{2}\right) \mathbf{X}\right] \geq \gamma_{2} \sigma_{S 2}^{2}, \\
& \mathbf{X}(i, i) \leq P_{R}^{i} / \mathbf{D}(i, i), i=1, \cdots, K, \\
& \mathbf{X} \succeq 0 .
\end{array}
$$

If it is feasible, we have $r \leq r^{*}$ and the corresponding rate is achievable. Otherwise, we have $r>r^{*}$ and the corresponding rate is not achievable. Based on this observation, we apply bi-section search over $r$ to solve the problem in (75), where we solve a convex feasibility problem of (80) at each step. We start with an interval $\left[0, r_{\max }\right]$ that contains the optimal value $r^{*}$ where $r_{\max }$ can be obtained in a similar way as that for the sum-power constrained case, and run the following algorithm.

\section{Algorithm 2}

- Initialize $r_{l o w}=0, r_{u p}=r_{\max }$.

- Repeat

1) Set $r \leftarrow \frac{1}{2}\left(r_{\text {low }}+r_{\text {up }}\right)$.

2) Solve the feasibility problem given by (80)-83) with given $r$.

3) Update $r$ : If the problem is feasible, set $r_{\text {low }}=r$; otherwise, $r_{u p}=r$.

- Until $r_{u p}-r_{\text {low }}<\epsilon$. Then $r^{*}=r_{\text {low }}$.

1) Rank-one solution based on randomization: Similar to the sum-power constrained case, the solution of $\mathbf{X}$ at the end of Algorithm 2, denoted as $\mathbf{X}_{o p t}$, may not be rank-one. However, since there are $K+2$ linear constraints here, we cannot apply the rank-one decomposition technique in [18], which require the number of linear constraints to be less than or equal to 3. Fortunately, various techniques have been developed [19] to generate good rankone approximate solutions to the original problem 22. One such efficient approach is based on randomization [19]: using $\mathbf{X}_{\text {opt }}$ to randomly generate a set of candidate weight vectors, $\left\{\mathbf{w}_{l}\right\}$, from which the "best" solution for the beamforming vector $\mathbf{w}$ is selected. There are three ways of generating $\left\{\mathbf{w}_{l}\right\}$ as presented in [19]. In order to satisfy the individual power constraint, we adopt the routine named randB in [19]. Specially, let $\mathbf{e}_{l}$ be the vector whose elements are independent random variables uniformly distributed on the unit circle in the complex plane, i.e., its $i$-th element $\left[\mathbf{e}_{l}\right]_{i}=e^{j \theta_{l, i}}$, where $\theta_{l, i}$ 's are independent and uniformly distributed over $[0,2 \pi)$. We choose $\mathbf{w}_{l}$ such

\footnotetext{
${ }^{2}$ The randomization technique only provides approximate solutions. Hence, the corresponding rate region is not exact.
} 
that its $i$-th element $\left[\mathbf{w}_{l}\right]_{i}=\sqrt{\left[\mathbf{X}_{o p t}\right]_{i i}}\left[\mathbf{e}_{l}\right]_{i}$. As we see, $\left|\left[\mathbf{w}_{l}\right]_{i}\right|^{2}=\left[\mathbf{X}_{o p t}\right]_{i i}$; hence the individual power constraint can be satisfied.

For each $\mathbf{X}^{(l)}=\mathbf{w}_{l} \mathbf{w}_{l}^{H}$, we associate each $\mathbf{w}_{l}$ with a value $v\left(\mathbf{w}_{l}\right)$,

$$
\begin{aligned}
v\left(\mathbf{w}_{l}\right)= & \max \left(1-\operatorname{tr}\left[\left(\frac{P_{S 1} \mathbf{F}_{1}}{\gamma_{2} \sigma_{S 2}^{2}}-\frac{\mathbf{A}_{2}}{\sigma_{S 2}^{2}}\right) \mathbf{w}_{l} \mathbf{w}_{l}^{H}\right],\right. \\
& \left.1-\operatorname{tr}\left[\left(\frac{P_{S 2} \mathbf{F}_{2}}{\gamma_{1} \sigma_{S 1}^{2}}-\frac{\mathbf{A}_{1}}{\sigma_{S 1}^{2}}\right) \mathbf{w}_{l} \mathbf{w}_{l}^{H}\right]\right),
\end{aligned}
$$

which reflects how much the constraints are violated. The "best" weight vector among the candidate vectors is the one that has the minimum $v\left(\mathbf{w}_{l}\right)$, i.e.,

$$
\begin{aligned}
l^{*} & =\arg \min _{l} v\left(\mathbf{w}_{l}\right), \\
\mathbf{w}^{*} & =\mathbf{w}_{l^{*}} .
\end{aligned}
$$

\section{Sub-optimal Schemes}

In this section, we propose some suboptimal schemes with lower complexity for implementation than the optimal ones established in the previous sections.

\section{A. Reciprocal Channel Case}

In the reciprocal channel case, at first the transmit phases $\theta_{i}$ 's at the relays are matched to the channels as $\theta_{i}=-\left(\angle h_{1, i}+\angle h_{2, i}\right)$. Then with the sum-power constraint, we propose the sub-optimal equal power beamforming scheme where each relay transmits with equal power. With the individual-power constraints, we propose the maxpower beamforming scheme where each relay transmits with its maximum power.

1) Equal-power beamforming: All the $K$ relay nodes transmit with the same power $P_{R} / K ; \theta_{i}$ 's and $x_{i}$ 's for $i=1, \cdots, K$, are given as:

$$
\begin{aligned}
\theta_{i} & =-\left(\angle h_{1, i}+\angle h_{2, i}\right), \\
x_{i} & =\sqrt{\frac{P_{R}}{K\left(P_{S 1}\left|h_{1, i}\right|^{2}+P_{S 2}\left|h_{2, i}\right|^{2}+\sigma_{i}^{2}\right)}} .
\end{aligned}
$$

2) Max-power beamforming: Each relay transmits with its maximum allowable power $P_{R, i} ; \theta_{i}$ 's and $x_{i}$ 's for $i=1, \cdots, K$, are given as:

$$
\begin{aligned}
\theta_{i} & =-\left(\angle h_{1, i}+\angle h_{2, i}\right), \\
x_{i} & =\sqrt{\frac{P_{R, i}}{P_{S 1}\left|h_{1, i}\right|^{2}+P_{S 2}\left|h_{2, i}\right|^{2}+\sigma_{i}^{2}}} .
\end{aligned}
$$


These sub-optimal schemes enjoy implementation simplicity since each relay only requires the local channel information $h_{1, i}$ and $h_{2, i}$ to decide the transmit phase and $x_{i}$.

\section{B. Non-reciprocal Channel Case}

For the non-reciprocal channel case, since the transmit phase cannot be matched to the two-directional channels simultaneously, we propose a sub-optimal scheme that greedily chooses the transmit phases. Specifically, each relay chooses the transmit phase to be either $\angle h_{1, i}+\angle h_{2, i}^{r}$ or $\angle h_{2, i}+\angle h_{1, i}^{r}$, whichever maximizes its own contribution to the overall SNRs at S1 or S2 without considering any other relays' contributions, i.e., we pick one of the above two phases that maximizes the following quantity:

$$
\max \left(\frac{x_{i}^{2} P_{S 2}\left|h_{2, i} h_{1, i}^{r} e^{j \theta_{i}}\right|^{2}}{\sigma_{S 1}^{2}+x_{i}^{2}\left|h_{1, i}^{r}\right|^{2} \sigma_{i}^{2}}, \frac{x_{i}^{2} P_{S 1}\left|h_{1, i} h_{2, i}^{r} e^{j \theta_{i}}\right|^{2}}{\sigma_{S 2}^{2}+x_{i}^{2}\left|h_{2, i}^{r}\right|^{2} \sigma_{i}^{2}}\right)
$$

where $x_{i}$ is the transmit amplitude. To determine $x_{i}$ 's, we adopt equal-power beamforming for the sum-power constraint case and max-power beamforming for the individual-power constraint case, which are given in (89) and (91), respectively.

\section{NumericAl Results}

In the section, we present numerical results to quantify the achievable rate region for the two-way relay network with collaborative beamforming. We assume that the relay cluster consists of 5 nodes; the channel coefficients $h_{1, i}$ and $h_{1, i}^{r}, i=1, \cdots, K$, are independent CSCG variables with distribution $\mathcal{C N}(0,1)$; the channel coefficients $h_{2, i}$ and $h_{2, i}^{r}, i=1, \cdots, K$, are also independent and distributed as $\mathcal{C N}(0,1)$. The noises at the relays and source nodes are assumed to have unit variance in the simulations. We change $\mu$ from 0 to 1 with step 0.1 and obtain 11 Pareto boundary points. For each point, we run 100 channel realizations to measure the expected performance. We then do convex hulling over these points.

First, we investigate the achievable rate region. Both reciprocal channel and non-reciprocal channel cases are discussed under a sum-power constraint and individual-power constraints, respectively. When the channel is reciprocal, we set $h_{1, i}=h_{1, i}^{r}$ and $h_{2, i}=h_{2, i}^{r}$ for $i=1, \cdots, K$. As shown in Fig. 2] the solid curves represent the reciprocal channel cases, with the outer one denoting the sum-power constraint case and the inner one denoting the individual-power constraints case; the dashed curves represent the non-reciprocal channel cases, with the outer one denoting the sum-power constraint case and the inner one denoting the individual-power constraints case. For the sum-power constraint case, the relay power $P_{R}=10 \mathrm{~W}$ while the transmit powers $P_{S 1}=P_{S 2}=1 \mathrm{~W}$. For the individual-power constraints case, the relay power constraints are given as 2.5, 3, 0.5, 1, $3 \mathrm{~W}$ (noises are assumed to have unit power in Watt), which is summed up to $10 \mathrm{~W}$. We use CVX, a Matlab-based optimization software 


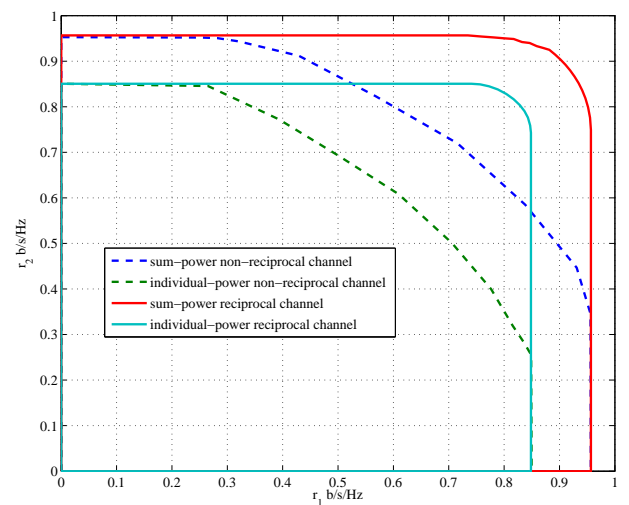

Fig. 2: Achievable rate regions for reciprocal and non-reciprocal channels with sum-power constraint and individual-power constraint, respectively. Transmitter powers: $P_{S 1}=P_{S 2}=1 \mathrm{~W}$, relay network power $P_{R}=10 \mathrm{~W}$ (sum-power constraint), $\mathbf{P}_{R}=$ $[2.5,3,0.5,1,3] \mathrm{W}$ (individual power constraint).

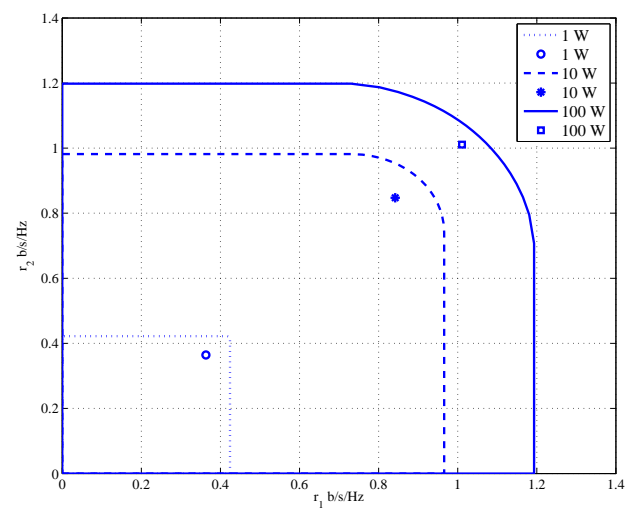

Fig. 3: Achievable rate regions for reciprocal channel case under a sum-power constraint, network total power are $0,10,100$ $\mathrm{W}$, using equal-power beamforming as sub-optimal scheme.

[20], to solve the SDP problems. As we see in Fig. 2] due to the symmetry of the transmit powers and channel statistics, the achievable rate region $\mathcal{O}$ is symmetric. When $P_{S 2}=0$, the rate pairs collapse to the segment on the horizontal axis, which corresponds to the achievable rate for a one-way relay network where only S1 transmits. Moreover, the rate region for the individual-power constraint case is smaller than that for the sum-power constraint case. This is quite intuitive since the individual-power constraint is stricter than the sum-power constraint.

In Fig. 2, we also compare the rate regions for the reciprocal and non-reciprocal channel cases under the same power constraint assumption. As we can see, the maximum rate for $\mathrm{S} 1$ in the reciprocal channel case is the same as the one in the non-reciprocal channel case. This is because such a maximum rate is obtained by optimizing the one-way link from S1 to S2 without considering the link from S2 to S1. Since the one-way link from S1 to S2 consists of $\mathbf{h}_{1}$ and $\mathbf{h}_{2}^{r}$, whether $\mathbf{h}_{1}^{r}=\mathbf{h}_{1}$ or not does not affect the statistics of the one-way link from S1 to S2. The same argument holds for maximum rate at S2. We also observe that the rate region for the reciprocal channel case is larger than that in the non-reciprocal channel case given the same settings of powers and noises. The reason is 


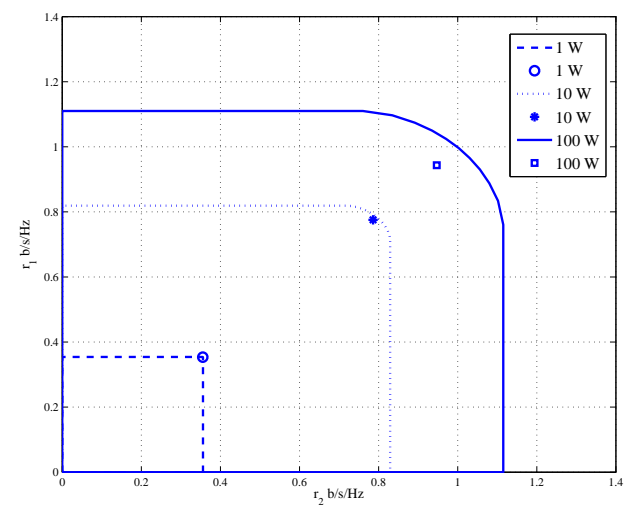

Fig. 4: Achievable rate regions for reciprocal channel case under individual-power constraints, network total power are $0,10,100 \mathrm{~W}$, using max-power beamforming as sub-optimal scheme.

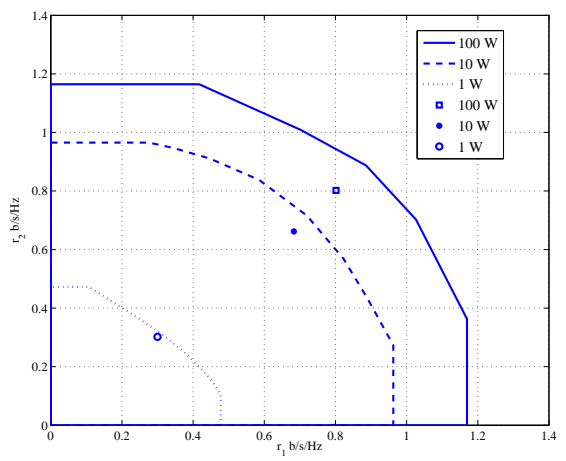

Fig. 5: Achievable rate regions for non-reciprocal channel case under a sum-power constraint, network total power are 0,10,100 W, using equal-power beamforming as sub-optimal scheme.

that we can match the beamforming phase to the overall channel phase (i.e., $\angle w_{i}=\angle h_{1, i}+\angle h_{2, i}, i=1, \cdots, K$.) in the reciprocal channel case, while we are not able to do so in the non-reciprocal channel case. Therefore, TDD based system is more favorable in terms of the achievable rate region if the channel coherence time is larger than one operation period and the transmit-receive chain calibration [21] can be properly done. Besides the rate region, the amount of information needs to be broadcast by the control center is significantly different. In the reciprocal channel case, the control center only needs to broadcast one scalar at each time slot. However, in the non-reciprocal channel case, the control center needs to broadcast the beamforming vector, which is a complex vector of dimension $K$.

Second, we investigate the performance of the sub-optimal schemes in relative to the maximum achievable rate regions. As we see in Fig. 3 for reciprocal channels case under the sum-power constraint, the rate pairs achieved by the equal-power beamforming scheme, denoted as single points, are strictly sub-optimal. On the contrary, as shown in Fig. 4 for the individual-power constraints case, the rate pair achieved by max-power beamforming gets closer to 


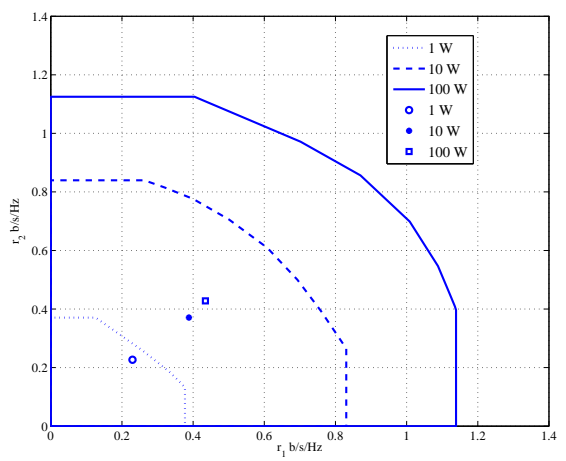

Fig. 6: Achievable rate regions for non-reciprocal channel case under individual-power constraints, network total power are 0,10,100 W, using maximum-power beamforming as sub-optimal scheme.

the boundary when the power budget is reduced 3 In Fig. 5 and Fig. 6, we consider the non-reciprocal channels and show the performance of equal-power beamforming and max-power beamforming with greedy phase selection as given in (92). The performance of both equal-power beamforming and max-power beamforming schemes degrades as $P_{R}$ increases. Thereby, the sub-optimal schemes for the non-reciprocal channel case works well only when $P_{R}$ is small.

\section{CONCLUSION}

In this paper, we considered the two-way relay networks with collaborative beamforming and investigated the achievable rate region, which is defined as the convex hull of all achievable rate pairs. We studied both the reciprocal and non-reciprocal channel cases. In the reciprocal channel case, we characterized the rate region when the relay cluster is subject to either a sum-power constraint or individual-power constraints, respectively. It was shown that we could characterize the whole achievable rate region via the Pareto-optimal beamforming vectors obtained from solving a sequence of WSISMin problems. Furthermore, we derived the closed-form solutions for those optimal beamforming vectors and consequently proposed partially distributed algorithms to implement the optimal beamforming, where each relay node only needs its own local channel information and one global scalar sent from the control center. For the non-reciprocal channel case, we used the rate-profile approach to compute the Pareto-optimal beamforming vectors. When the relay cluster is subject to a sum-power constraint, we computed the optimal beamforming vector via solving a sequence of relaxed SDP power minimization problems followed by a special rank-one reconstruction. When the relay cluster is subject to individual-power constraints, we solved a sequence of relaxed SDP feasibility problems and the rank-one solution is obtained by randomization techniques. From the numerical results, we found that the achievable rate region is larger in the reciprocal channel case than

\footnotetext{
${ }^{3}$ We set the individual powers $\mathbf{P}_{R}=[2.5,3,0.5,1,3] \mathrm{W}$ with total power equal to $10 \mathrm{~W}$. When total power is changed to $1 \mathrm{~W}$ and 100 $\mathrm{W}$, we scale the vector proportionally.
} 


$$
\begin{aligned}
\frac{d p^{2}(y)}{d y^{2}} & =\frac{d p^{\prime}(y) / d x}{d y / d x} \\
& =-\frac{x+x^{2}}{\ln 2}\left[\frac{q^{\prime \prime}(x)\left(x+x^{2}\right)}{q(x)+q(x)^{2}}+q^{\prime}(x)\left(\frac{(1+2 x)\left(q(x)+q(x)^{2}\right)-\left(q^{\prime}(x)+2 q(x) q^{\prime}(x)\right)\left(x+x^{2}\right)}{q(x)+q(x)^{2}}\right)\right]>0 .
\end{aligned}
$$

that in the non-reciprocal channel case. Hence, TDD-based relaying scheme is more favorable for the two-way relay network with collaborative beamforming.

\section{APPENDICES}

\section{A. Proof of Proposition 1}

Proof: We will show this by contradiction. Assume $(a, b) \in \mathcal{B}$ but $\mathcal{U}((a, b)) \notin \mathcal{P}$. Then we can find another point $(c, d) \in \mathcal{R}$ such that $c>1 / 2 \log _{2}(1+1 / a)$ and $d>1 / 2 \log _{2}(1+1 / b)$. According to the definition of $\mathcal{I}$, the point $\left(\frac{1}{2^{2 c}-1}, \frac{1}{2^{2 d}-1}\right) \in \mathcal{I}$. Thus, there exists a point in $\mathcal{I}$ such that $\frac{1}{2^{2 c}-1}<a$ and $\frac{1}{2^{2 d}-1}<b$, which contradicts the assumption that $(a, b)$ is a Pareto optimal point. Hence $\mathcal{U}(\mathcal{B}) \subseteq \mathcal{P}$. The converse that $\mathcal{U}(\mathcal{B}) \supseteq \mathcal{P}$ can also be proven in the similar way. Therefore, $\mathcal{P}=\mathcal{U}(\mathcal{B})$.

\section{B. Proof of Proposition 2}

In order to prove Proposition 2, we first introduce the following two lemmas.

Lemma 1: Suppose $q(x)$ is a positive, decreasing, and linear function with $x>0$. The bijective mapping $\mathcal{U}$ maps $(x, q(x))$ to $(y, p(y))$; then $p(y)$ is a non-negative, decreasing, and convex function.

Proof: Let $y=\log _{2}(1+1 / x)$ and $p(y)=\log _{2}(1+1 / q(x))$ be an implicit function of $y$, where $x>0$. Since $q(x)$ is positive, decreasing, and linear, we have $q(x)>0, q^{\prime}(x)<0, q^{\prime \prime}(x)=0$, and hence $p(y) \geq 0$. The first-order derivative of $p(y)$ is

$$
\begin{aligned}
p^{\prime}(y) & =\frac{d p(y) / d x}{d y / d x} \\
& =q^{\prime}(x) \frac{x+x^{2}}{q(x)+q(x)^{2}}<0 .
\end{aligned}
$$

The second-order derivative is given by 93 , which is positive.

Thus, $p(y)$ is a convex function of $y$.

According to the above lemma, the line segment $\overline{Q_{1} Q_{2}}$ in Fig. $\overline{7(a)}$ is mapped to a convex curve $\widehat{Q_{1}^{\prime} Q_{2}^{\prime}}$ in Fig. 7(b) by $\mathcal{U}$. In addition, it is easy to see that $\overline{Q_{1} Q_{2}}+\mathbf{K} \mapsto \widehat{Q_{1}^{\prime} Q_{2}^{\prime}}-\mathbf{K}$, i.e., any point above $\overline{Q_{1} Q_{2}}$ (for example, $P$ in Fig. 7(a) will be mapped to be a point below $\widehat{Q_{1}^{\prime} Q_{2}^{\prime}}$ (i.e., $P^{\prime}$ in Fig. 7(b)]. 


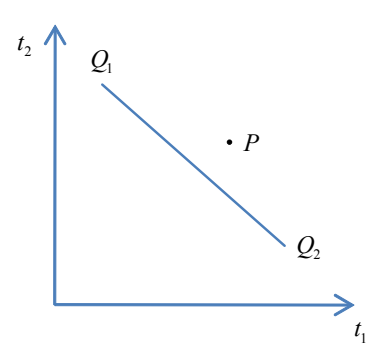

(a) A straight line in $\mathcal{I}$.

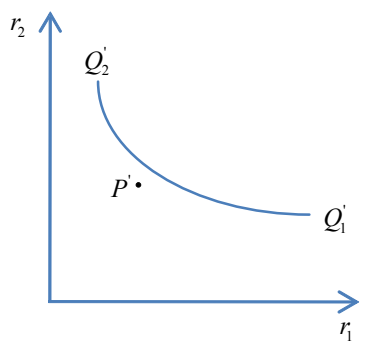

(b) The image of $\overline{Q_{1}^{\prime} Q_{2}^{\prime}}$ in $\mathcal{R}$.

Fig. 7: Illustration of Lemma 1. Mapping a straight line in $\mathcal{I}$ to a convex curve in $\mathcal{R}$.

Lemma 2: Let a point $\left(q_{1}, q_{2}\right) \in \mathbf{b d}(\mathcal{I}) \backslash \mathcal{S}(\mu, \mathcal{I})$, where $\mathbf{b d}(\mathcal{I})$ denotes the boundary of region $\mathcal{I}$. If $q_{1}=$ $\lambda t_{1}+\bar{\lambda} s_{1}$, where $\left(t_{1}, t_{2}\right),\left(s_{1}, s_{2}\right) \in \mathcal{S}(\mu, \mathcal{I})$ and $\left(t_{1}, t_{2}\right) \neq\left(s_{1}, s_{2}\right)$, we have $q_{2}>\lambda t_{2}+\bar{\lambda} s_{2}$, i.e., the point $\left(q_{1}, q_{2}\right)$ is above the line segment connecting $\left(t_{1}, t_{2}\right)$ and $\left(s_{1}, s_{2}\right)$.

Proof: We show this by contradiction. Suppose $\mathcal{S}(\mu, \mathcal{I})$ has more than one elements for a given $\mu$, such that $\left(t_{1}, t_{2}\right),\left(s_{1}, s_{2}\right) \in \mathcal{S}(\mu, \mathcal{I})$, and $\left(t_{1}, t_{2}\right) \neq\left(s_{1}, s_{2}\right)$. According to the definition of $\mathcal{S}(\mu, \mathcal{I})$ given by (18), we have $\mu t_{1}+\bar{\mu} t_{2}=\mu s_{1}+\bar{\mu} s_{2}=m$, where $m$ is the minimum value of the weighted sum for a given $\mu$ over all points in $\mathcal{I}$. If $q_{1}=\lambda t_{1}+\bar{\lambda} s_{1}$ and $q_{2} \leq \lambda t_{2}+\bar{\lambda} s_{2}$, we have

$$
\begin{aligned}
\mu q_{1}+\bar{\mu} q_{2} & \leq \mu\left(\lambda t_{1}+\bar{\lambda} s_{1}\right)+\bar{\mu}\left(\lambda t_{2}+\bar{\lambda} s_{2}\right) \\
& =\lambda\left(\mu t_{1}+\bar{\mu} t_{2}\right)+\bar{\lambda}\left(\mu s_{1}+\bar{\mu} s_{2}\right) \\
& =m
\end{aligned}
$$

If $\mu q_{1}+\bar{\mu} q_{2}<m$, it contradicts that $m$ is the minimum value of the weighted sum for the given $\mu$; If $\mu q_{1}+\bar{\mu} q_{2}=m$, it contradicts that $\left(q_{1}, q_{2}\right)$ is not in $\mathcal{S}(\mu, \mathcal{I})$. Therefore, the lemma holds.

According to the above lemma, for a given $\mu$, if $\mathcal{S}(\mu, \mathcal{I})$ has more than one elements, the set of boundary points $\left\{\left(q_{1}, q_{2}\right):\left(q_{1}, q_{2}\right) \in \mathbf{b d}(\mathcal{I}) \backslash \mathcal{S}(\mu, \mathcal{I}), s_{1}<q_{1}<t_{1},\left(s_{1}, s_{2}\right),\left(t_{1}, t_{2}\right) \in \mathcal{S}(\mu, \mathcal{I})\right\}$ must be above the line segment connecting $\left(s_{1}, s_{2}\right)$ and $\left(t_{1}, t_{2}\right)$; and hence are not attainable by solving WSISMin. This is true for all $\mu$ 's; hence if a boundary point is not attainable by solving WSISMin, it must be above a line segment connecting two particular points in $\mathcal{S}(\mu, \mathcal{I})$ for some $\mu$. With the above two lemmas, we are ready to prove Proposition 2 as follows.

\section{Proof of Proposition 2}

Proof: First we define

$$
\Delta=\{\mu: \mathcal{S}(\mu, \mathcal{I}) \text { has more than one elements }\},
$$

and let $l_{\mu}$ be the line segment (e.g., $\overline{A B}$ in Fig. $8(\mathrm{a})$ with two end points from $\mathcal{S}(\mu, \mathcal{I})$ for $\mu \in \Delta$ (e.g., points $A$ and $B$ in $8(\mathrm{a})$. According to Lemma 2, the boundary points that are not attainable by solving WSISMin, denoted as 


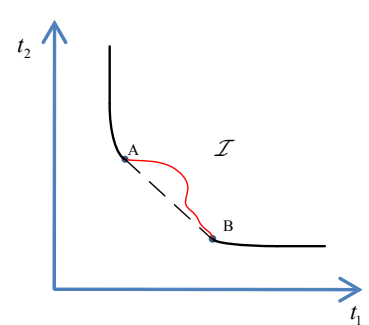

(a) Inverse-SNR region

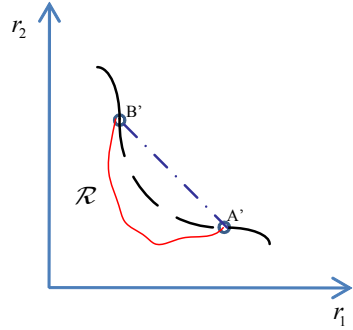

(b) Rate region

Fig. 8: Inverse-SNR region and corresponding rate and region.

$\operatorname{bd}(\mathcal{I}) \backslash \mathcal{S}(\mathcal{I})$ (here referring to curve $\widehat{A B}$ in Fig. $8($ a) $)$, must be above $l_{\mu}$ 's, i.e., bd $(\mathcal{I}) \backslash \mathcal{S}(\mathcal{I}) \subseteq \bigcup_{\mu \in \Delta}\left(l_{\mu}+\mathbf{K}\right)$; and it follows that $\mathcal{U}(\mathbf{b d}(\mathcal{I}) \backslash \mathcal{S}(\mathcal{I})) \subseteq \mathcal{U}\left(\bigcup_{\mu \in \Delta}\left(l_{\mu}+\mathbf{K}\right)\right)$. According to Lemma $1 \mathcal{U}\left(\bigcup_{\mu \in \Delta}\left(l_{\mu}+\mathbf{K}\right)\right) \subseteq \bigcup_{\mu \in \Delta}\left(\mathcal{U}\left(l_{\mu}\right)-\mathbf{K}\right)$, where $\mathcal{U}\left(l_{\mu}\right)$ is a convex curve (e.g., here $\mathcal{U}\left(l_{\mu}\right)$ refers to the dashed convex curve $\widehat{A^{\prime} B^{\prime}}$ in Fig. $8(\mathrm{~b})$. Let $\tilde{l}_{\mu}$ be a line segment (i.e., the dot-dashed line segment $\overline{A^{\prime} B^{\prime}}$ in Fig. $8($ b) $]$ that connects the two end points of the convex curve $\mathcal{U}\left(l_{\mu}\right)$. Due to the convexity of $\mathcal{U}\left(l_{\mu}\right)$, we have $\mathcal{U}\left(l_{\mu}\right)-\mathbf{K} \subseteq \tilde{l}_{\mu}-\mathbf{K}$ and hence $\mathcal{U}(\mathbf{b d}(\mathcal{I}) \backslash \mathcal{S}(\mathcal{I})) \subseteq \bigcup_{\mu \in \Delta}\left(\tilde{l}_{\mu}-\mathbf{K}\right)$.

Notice $\bigcup_{\mu \in \Delta}\left(\tilde{l}_{\mu}+\mathbf{K}\right)$ is sufficient for constructing $\mathcal{O}$ by convex hulling. Therefore, $\mathcal{U}(\mathbf{b d}(\mathcal{I}) \backslash \mathcal{S}(\mathcal{I}))$ or $\mathbf{b d}(\mathcal{I}) \backslash \mathcal{S}(\mathcal{I})$ is not necessary for constructing $\mathcal{O}$. Since $\mathcal{B} \subseteq \mathbf{b d}(\mathcal{I})$, the set $\mathcal{B} \backslash \mathcal{S}(\mathcal{I})$ is also not necessary for constructing $\mathcal{O}$.

\section{REFERENCES}

[1] J. Laneman, D. Tse, and G. Wornell, "Cooperative diversity in wireless networks: Efficient protocols and outage behavior," IEEE Trans. Inf. Theory, vol. 50, no. 12, pp. 3062-3080, Dec. 2004.

[2] T. Cover and A. E. Gamal, “Capacity theorems for the relay channel," IEEE Trans. Inf. Theory, vol. 25, no. 5, pp. 572-584, Sep. 1979.

[3] G. Kramer, M. Gastpar, and P. Gupta, "Cooperative strategies and capacity theorem for relay networks," IEEE Trans. Inf. Theory, vol. 51, no. 9, pp. 3037-3063, Sep. 2005.

[4] M. Janani, A. Hedayat, T. E. Hunter, and A. Nosratinia, "Coded cooperation in wireless communications: Spacectime transmission and iterative decoding," IEEE Trans. Signal Process., vol. 52, no. 2, pp. 362-371, Feb. 2004.

[5] B. Khoshnevis, W. Yu, and R. Adve, "Grassmannian beamforming for MIMO amplify-and-forward relaying," IEEE J. Sel. Areas Commun., vol. 26, no. 8, pp. 1397-1407, Oct. 2008.

[6] S. Cui, A. J. Goldsmith, and A. Bahai, "Energy-efficiency of MIMO and cooperative MIMO in sensor networks," IEEE J. Sel. Areas Commun., vol. 22, no. 6, pp. 1089-1098, Aug. 2004.

[7] V. Nassab, S. Shahbazpanahi, A. Grami, and Z.-Q. Luo, "Distributed beamforming for relay networks based on second order statistics of the channel state information,” IEEE Trans. Signal Process., vol. 56, no. 9, pp. 4306-4316, Sep. 2008.

[8] Y. Jing and H. Jafarkhani, "Network beamforming using relays with perfect channel information," IEEE Trans. Inf. Theory, vol. 55, no. 6, pp. 2499 - 2517, Jun. 2009.

[9] R. Zhang, Y.-C. Liang, C. Choy, and S. Cui, "Optimal beamforming for two-way multi-antenna relay channel with analogue network coding,” IEEE J. Sel. Areas Commun., vol. 27, no. 5, pp. 699-712, Jun. 2009.

[10] S. Katti, S. Gollakota, and D. Katabi, "Embracing wireless interference: Analog networking coding," Computer Science and Artificial Intelligence Laboratory Technical Report, MIT-CSAIL-TR-2007-012, Feb. 2007.

[11] T. J. Oechtering, R. F. Wyrembelski, and H. Boche, "Multiantenna bidirectional broadcast channels - optimal transmit strategies," IEEE Trans. Signal Process., vol. 57, pp. 1948 - 1958, May. 2009. 
[12] S. J. Kim, P. Mitran, and V. Tarokh, "Performance bounds for bi-directional coded cooperation protocols," IEEE Trans. Inf. Theory, vol. 54, no. 11, pp. 5235 - 5240, Nov. 2008.

[13] V. Havary-Nassab, S. Shahbazpanahi, and A. Grami, "Optimal distributed beamforming for two-way relay networks," IEEE Trans. Signal Process., vol. 58, no. 3, pp. 1238 - 1250, Mar. 2010.

[14] R. Vaze and R. W. Heath, "Optimal amplify and forward strategy for two-way relay channel with multiple relays," in Proc. IEEE Information Theory Workshop, Volos, Greece, Jun. 2009.

[15] S. Boyd and L. Vandenberghe, Convex Optimization. Cambridge, U.K.: Cambridge University Press, 2003.

[16] X. Tang and Y. Hua, "Optimal design of non-regenerative MIMO wireless relays," IEEE Trans. Wireless Commun., vol. 6, no. 4, pp. 1398-1407, Apr. 2007.

[17] R. A. Horn and C. A. Johnson, Matrix Analysis. Cambridge, U.K.: Cambridge University Press, 1990.

[18] Y. Huang and S. Zhang, “Complex matrix decomposition and quadratic programming,” Math. Oper. Res., vol. 32, no. 3, pp. 758 - 768, Aug. 2007.

[19] N. D. Sidiropoulos, T. N. Davidson, and Z.-Q. Luo, "Transmit beamforming for physical-layer multicasting," IEEE Trans. Signal Process., vol. 54, no. 6, pp. 2239 - 2252, Jun. 2006.

[20] M. Grant and S. Boyd, “CVX users' guide for CVX version 1.21 (build 782)," May. 2010. [Online]. Available: http://cvxr.com/.

[21] E. Biglieri, R. Calderbank, A. Constantinides, A. Goldsmith, A. Paulraj, and H. V. Poor, Fundamentals of Wireless Communication. New York: Cambridge University Press, 2007. 